\title{
Vanadium carbide films produced by plasma-assisted metal-organic chemical vapour deposition
}

\author{
H. BERNDT*', A.-Q. ZENG, H.-R. STOCK* and P. MAYR* \\ Guangzhou Research Institute of Non-ferrous Metals, Wushan, Guangzhou, China \\ * Stiftung Institut für Werkstofftechnik, Badgasteiner Str. 3, 2800 Bremen 33, Germany
}

\begin{abstract}
Vanadium carbide films as wear-resistant coatings produced by salt bath immersion at high temperatures have found wide technological application in manufacturing industries. In order to reduce the substrate temperature, the deposition of VC-films by plasma-assisted chemical vapour deposition using a metal-organic compound as precursor was investigated.

Bis(cyclopentadienyl)-vanadium, a purple solid compound showing a sufficient sublimation rate at relatively low evaporator temperatures, was used together with hydrogen and argon to deposite VC-films in a d.c. glow discharge. As one of the most important parameters the substrate temperature was varied from $200{ }^{\circ} \mathrm{C}$ to $400{ }^{\circ} \mathrm{C}$. The compound decomposed into films and gaseous by-products like $\mathrm{C}_{5} \mathrm{H}_{6}$ and other hydrocarbons which could be detected by quadrupole mass spectrometer. The deposition efficiency of the coatings was almost independent of the substrate temperature. It was established by means of EDX and XRD that cubic vanadium carbide coatings were obtained. The morphology of these layers was examined by scanning electron microscopy. In the entire temperature range dense, polycrystalline layers were created, whose hardness measured between 2200-3300 HV. With the increase of the substrate temperature the adhesive strength of the coatings increased, too.
\end{abstract}

\section{Introduction}

Vanadium carbide (VC) is a very hard material, showing adequate toughness and excellent resistance to wear and corrosion. Because of these properties, VC-films deposited on various substrates have found wide applications in manufacturing industries, especially for automotive parts, such as progressive dies involved with piercing, chamfering and embossing $/ 1 /$.

The deposition of these VC-films can be carried out either by chemical vapour deposition (CVD) or by salt bath immersion process. In a salt bath process, high temperature of $1000{ }^{\circ} \mathrm{C}$ is necessary for the formation of the VC-films, because the carbon of the coating is supplied by the substrate through diffusion $/ 1 /$. The elevated temperature in the process offers the advantage of good adherence of the films. As a disadvantage a post-heat treatment must be taken into account when steel substrates are coated. For chemical vapour deposition, vanadium chlorides are used as the starting source $/ 2 /$. Because of the thermal stability of the precursor the reduction of the chloride by hydrogen takes place only at temperatures above $900^{\circ} \mathrm{C}$.

When volatile metal-organic compounds (MOC) showing a high chemical reactivity are used as precursors in a CVD-process (metal-organic CVD, MOCVD), the deposition temperature for wear-resistant coatings can be significantly reduced $/ 3 /$, owing to the low bonding energy in the metal-organic molecules. In this work we have used bis(cyclopentadienyl)-vanadium $\left[\left(\mathrm{C}_{5} \mathrm{H}_{5}\right)_{2} \mathrm{~V}\right.$ or $\left.\mathrm{Cp}_{2} \mathrm{~V}\right]$ in a plasma-assisted chemical vapour 
deposition (PACVD) equipment to produce vanadium carbide films at substrate temperatures in the range of $200-400^{\circ} \mathrm{C}$. The suitability of the precursor for the coating of high speed steel (HSS) without any post heat treatment could be shown.

\section{Experimental}

A precursor suitable for plasma-assisted MOCVD must be volatile and sufficiently stable to be transported in the gas phase to the deposition zone. Bis(cyclopentadienyl)-vanadium is a purple compound that exists in solid state at room temperature. The crystals, which are sensitive to air and moisture, have a melting point of approximately $170^{\circ} \mathrm{C} / 4 /$. But the compound can be well volatized at an evaporator temperature of $60^{\circ} \mathrm{C}$ with a typical sublimation rate of $1 \mathrm{~g} / \mathrm{h}$. Compared with other metal-organic compounds $\left(\mathrm{C}_{5} \mathrm{H}_{5}\right)_{2} \mathrm{~V}$ is thermally stable and no decomposition occurs below $170^{\circ} \mathrm{C}$.

The deposition was performed in a vacuum system with a d.c. power supply for the excitation of the plasma (Fig. 1). Using mass flow meters the gases hydrogen and argon (purity of $99.999 \%$ ) were admitted to a heated evaporator which contained the metal-organic compound. By means of a by-pass it was possible to heat the vacuum chamber up and cool it down by letting in the carrier gases without the addition of the MOC. By varying the temperature of the evaporator the partial pressure of the vanadium compound in the carrier gas could be adjusted.

The chamber was evacuated by means of mechanical pumps and an oil diffusion pump. Using the additional heating the vessel was baked during evacuation to remove adsorbed water. When the base pressure of $5 \cdot 10^{-6}$ mbar was achieved, the reaction chamber was filled with argon and hydrogen and a d.c. glow discharge (c. $200 \mathrm{~W})$ was ignited to clean the steel substrates by sputtering. Because of the ion bombardement the substrates were heated up simultaneously. The substrate temperature was measured with thermocouples and controlled via the applied voltage. Once the required temperature was reached, the coating process was started by closing the by-pass and allowing the carrier gas to flow through the evaporator. During the plasma process steps the total pressure was kept constant at 2 mbar by the roots pump plus the rotary vane pump. The gas composition in the reaction chamber was measured by a quadrupole mass spectrometer. The signal of light hydrocarbons which were produced in the plasma reaction could be used to adjust the temperature of the evaporator.

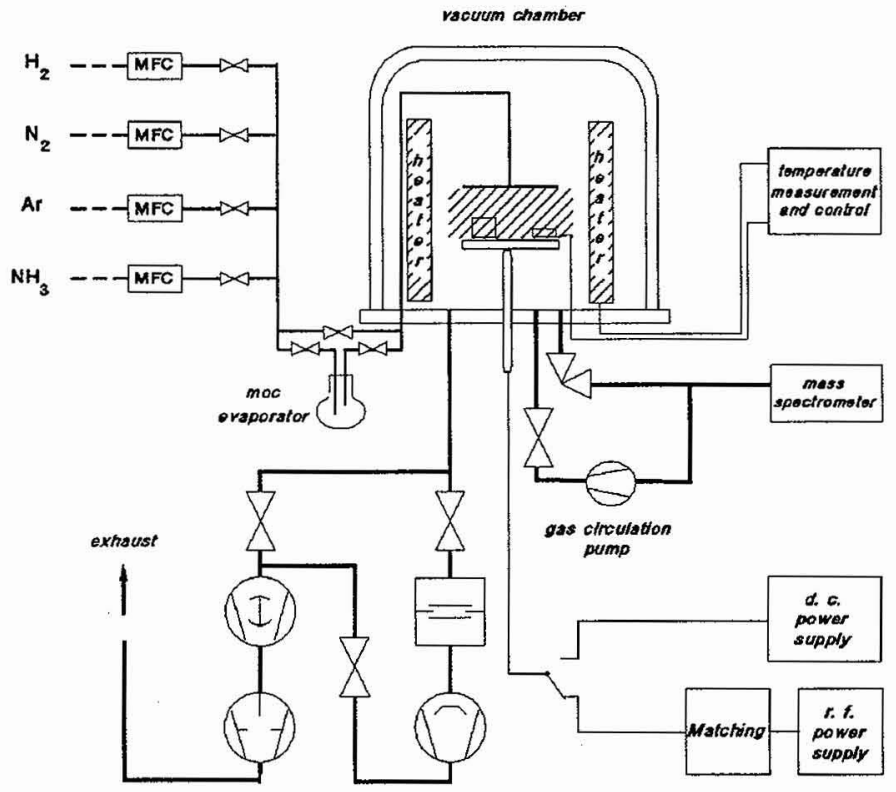

Fig. 1: Schematic drawing of the plasma-assisted CVD-unit 


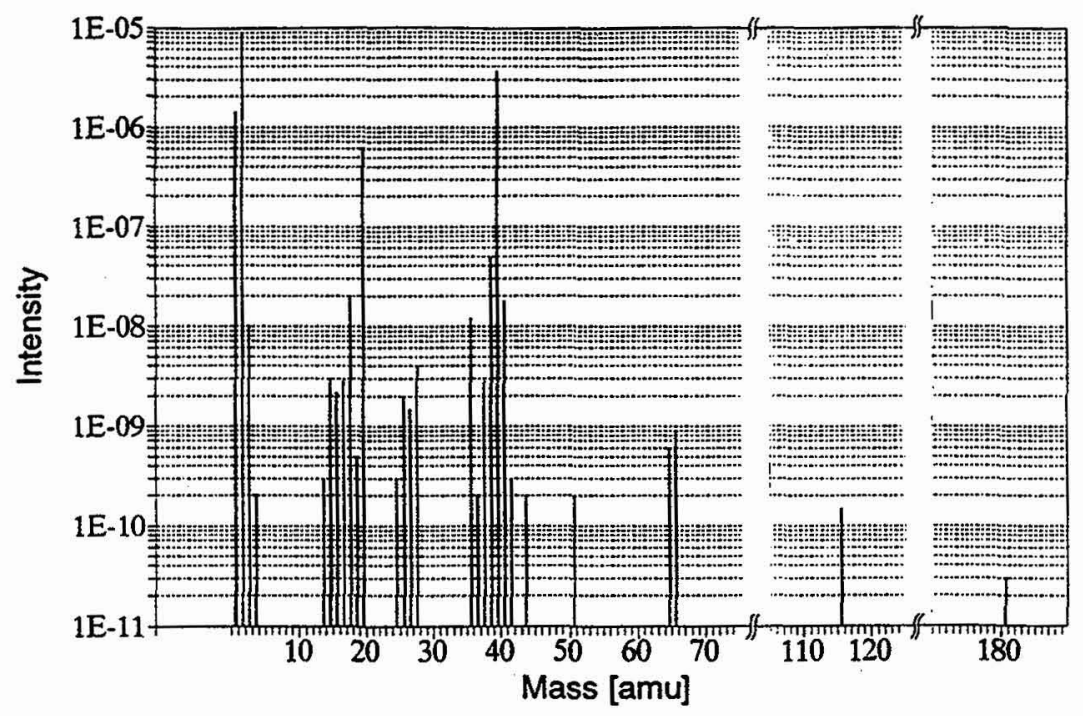

Fig. 2: Mass spectrum of the gas composition in the reaction chamber during the deposition process

\section{Results}

The precursor $\left(\mathrm{C}_{5} \mathrm{H}_{5}\right)_{2} \mathrm{~V}$ showed a high sublimation rate even at low evaporator temperatures. However, the sublimation rate varied in different experiments, even though the evaporator temperature was kept constant in every experiment. This phenomenon was considered to be related to the solid state of the precursor. Because of the little amount of MOC, which was filled in the evaporator, the grain size and the surface area of the compound played an important role in influencing the sublimation rate. However, it was possible to decompose the precursor in the glow discharge at substrate temperatures in the range of 200 to $400^{\circ} \mathrm{C}$. The coatings which were deposited in the entire temperature range exhibited a grey colour.

Figure 2 shows a typical mass spectrum of the gas composition in the reaction chamber. The peaks at $181\left(\mathrm{Cp}_{2} \mathrm{~V}\right), 116(\mathrm{CpV})$ and $51 \mathrm{amu}(\mathrm{V})$ are related to the undecomposed precursor and to precursor fragments, which are formed at the filament of the quadrupole mass spectrometer. Besides the carrier gases argon (36,38 and $40 \mathrm{amu}$ ) and hydrogen the products of the plasma reaction methane ( 15 and $16 \mathrm{amu}$ ), ethene and ethyne (25-28 amu) could be recorded. Due to the high partial pressure of argon and the abundance sensitivity of the mass spectrometer peaks appear at 39 and 41 amu, which have to be related to ${ }^{40} \mathrm{Ar}$, as could be shown by recording spectra without adding the MOC. Therefore a measurement of a small amount of hydrocarbons $\mathrm{C}_{3} \mathrm{H}_{x}$, which may exist, was not possible. From a comparison of the ratio of the peak intensities in the mass spectrum recorded during plasma deposition and in the mass spectrum of the undecomposed precursor it was obvious that cyclopentadiene $\mathrm{C}_{5} \mathrm{H}_{6}(65$ and $66 \mathrm{amu})$ is stable enough to be onty partly destroyed by the intense excitation in the glow discharge.

For the substrate temperature range the deposition rate of the coatings was in the range of $0.5-1.5 \mu \mathrm{m} / \mathrm{h}$, depending on the sublimation rate. To analyze the dependence of the deposition rate on the substrate temperature, the influence of the sublimation rate of the precursor has to be removed. Therefore we took the deposition efficiency as a criterion, which is defined as the ratio of the vanadium mass deposited in the coating to the vanadium mass in the metal-organic compound, which is consumed during the deposition process. The consumption of MOC is determined via the weight loss of the evaporator, while the vanadium mass deposited in the coating is calculated out of the film thickness taking into consideration the deposition on the samples and on the electrode. Figure 3 shows the deposition efficiency in the temperature range 


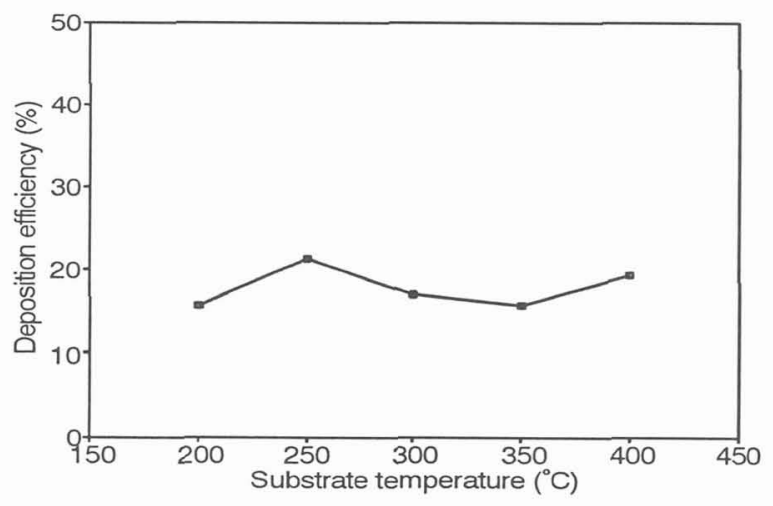

Fig. 3: Deposition efficiency of coatings as a function of the substrate temperature.
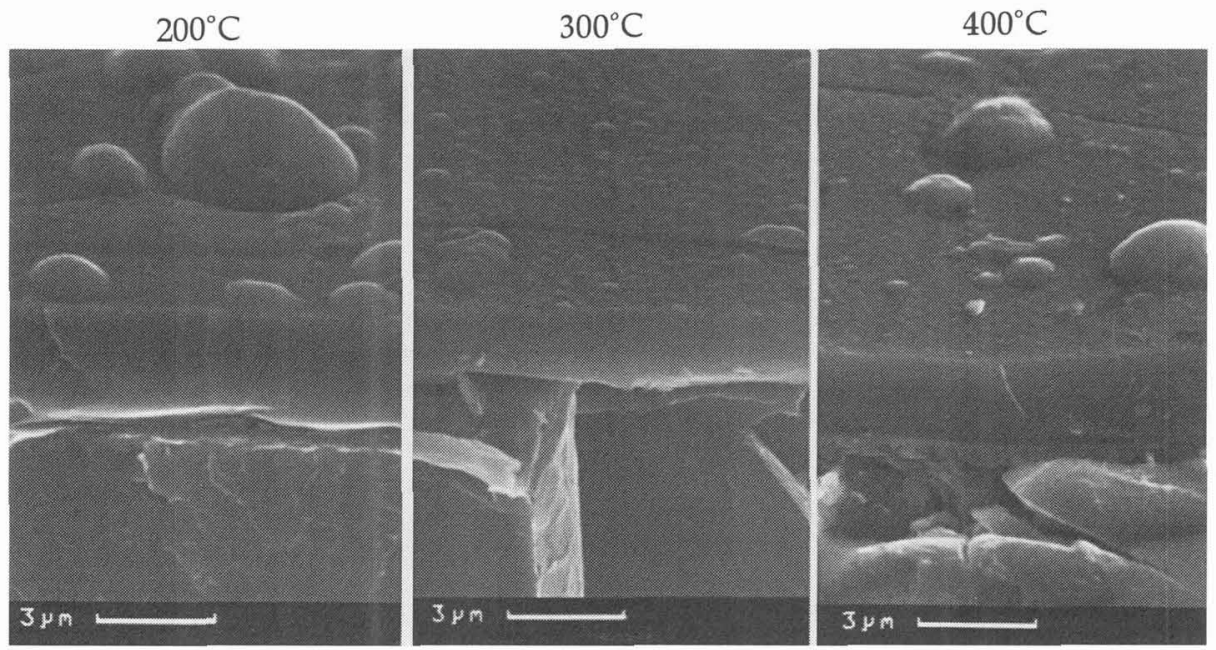

Fig. 4: Fractured cross-sections of VC-coatings, deposited at different substrate temperatures.

of 200 to $400{ }^{\circ} \mathrm{C}$. The flat curve indicates that the deposition efficiency is almost independent of the substrate temperature.

The morphology of the coatings was examined at the fractured cross-sections through the coatings using scanning electron microscopy. Figure 4 shows three micrographs of the coatings which were deposited at different substrate temperatures. All of the coatings exhibit a homogeneous dense structure on the cross-sections. Grains or columnar structures, well known from other deposition techniques, are not recognizable. On the surface of the coatings nodules appear which have the same chemical composition as the bulk substance of the layer, as revealed by energy dispersive $X$-ray analysis (EDX).

The structure of the coating surface is also influenced by the roughness of the substrate. Figure 5 represents two micrographs of layers which are deposited at a substrate temperature of $350{ }^{\circ} \mathrm{C}$ on the $\mathrm{C} 45$ carbon steel and on an indexable tip made of high speed steel. Prior to deposition the former was ground with $\mathrm{SiC}$ while the latter was shot blasted. It can be seen that 


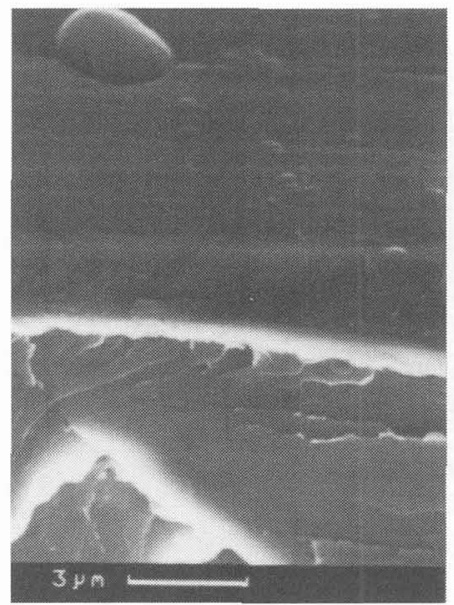

$\mathrm{b}$

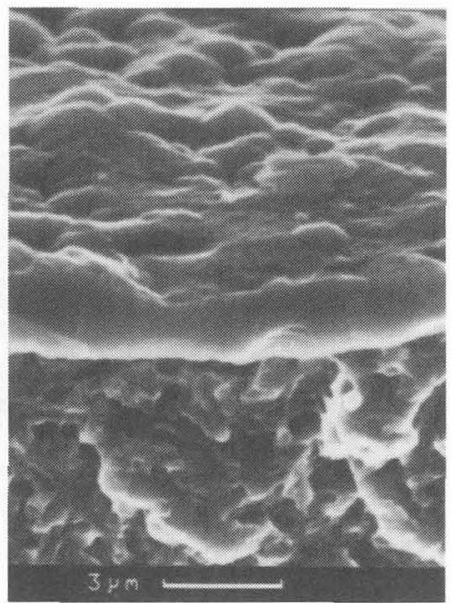

Fig. 5: Surface topography of the VC-coatings deposited at $350^{\circ} \mathrm{C}$ on: (a) plain carbon steel C45 (ground); (b) HSS cutting tool (shot blasted).

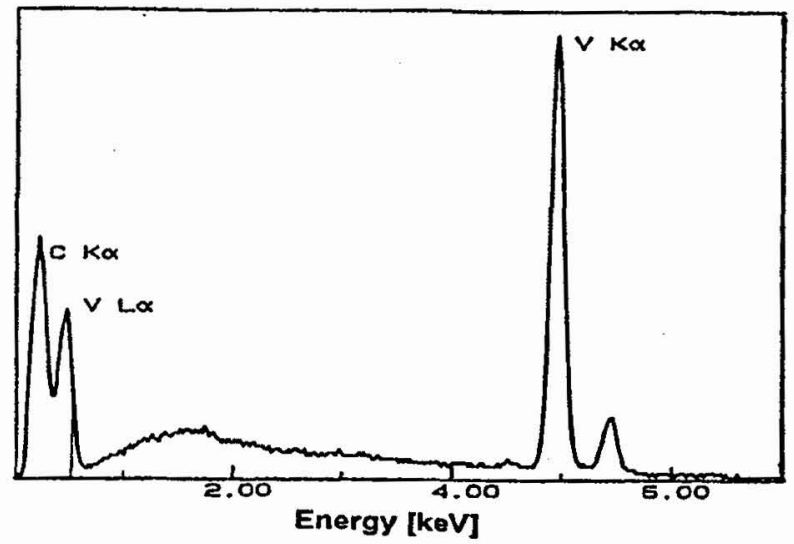

Fig. 6: EDX-spectrum of a VC-coating

the coating deposited on the cutting tool is much rougher than that on $\mathrm{C} 45$ and more nodules appear in the layer on HSS.

The chemical composition of the coatings was determined by means of EDX. Figure 6 shows a spectrum, obtained from a coating which was produced at a substrate temperature of $300^{\circ} \mathrm{C}$. Only vanadium and carbon are present in this layer. The chemical composition of the coatings, as revealed by EDX, is not dependent on the substrate temperature. Using X-ray diffraction $(\mathrm{XRD})$ it can be confirmed that vanadium and carbon exist in the crystal structure of the VCphase (Figure 7). The X-ray diffraction pattern was obtained with $\mathrm{Cu}-\mathrm{K}_{\alpha}$ radiation. The reflections in the spectrum are in accordance with the indexes of the cubic vanadium carbide $\{111\},\{200\},\{220\},\{311\},\{222\},\{400\},\{331\}$ and $\{420\}$ respectively. In addition to those reflections caused by vanadium carbide the sharp reflections of the bulk substrate material $\alpha$-Fe also appear in the diffraction pattern. 


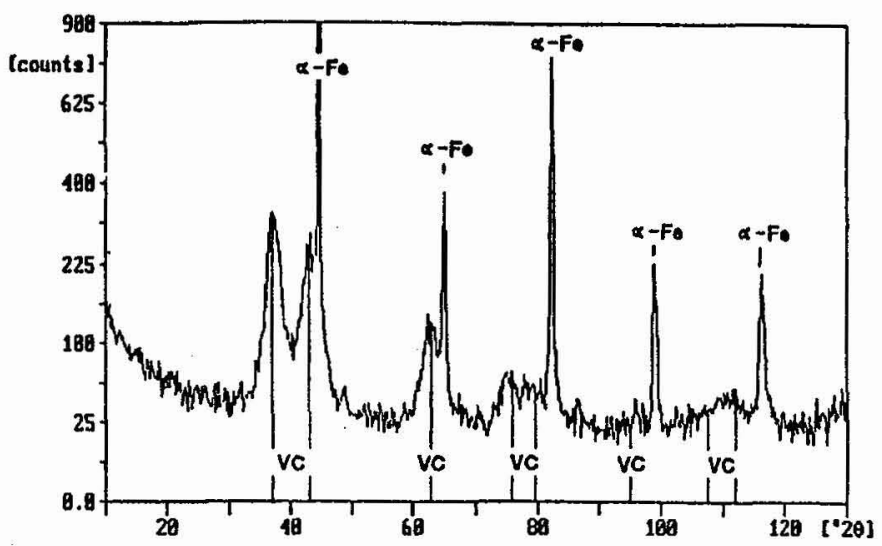

Fig. 7: X-ray diffraction pattern of a VC-coating on $\mathrm{C} 45\left(\mathrm{Cu}-\mathrm{K}_{\alpha}\right)$

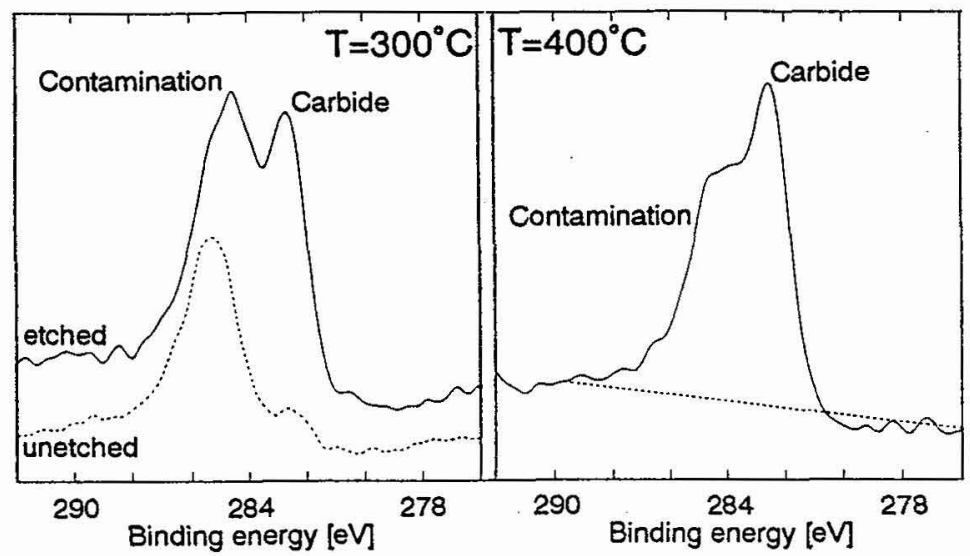

Fig. 8: XPS-data (C1s-peak), obtained from coatings deposited at $300^{\circ} \mathrm{C}$ and $400{ }^{\circ} \mathrm{C}$

One major problem in plasma-assisted MOCVD is the incorporation of organic fragments or carbon in the growing layer during the deposition /5, 6/, which can affect the properties of the coatings. In order to determine amorphous impurities, which cannot be registered by $X R D, X$ ray photoelectron spectroscopy (XPS) measurements are performed on the carbon 1s-peak to determine the kind of chemical bonding of the carbon in the layers. A comparison of the XPSdata obtained from coatings, which are deposited at 300 and $400^{\circ} \mathrm{C}$ respectively, is shown in Figure 8. On the unetched surface carbon is detected in the form of contamination (C-C or C-H bonds). After $10 \mathrm{~min}$ of argon sputter etching this contamination, which is adsorbed when the samples are handled in air, is removed and the properties of the bulk material could be analyzed. From the spectra it is obvious, that two kinds of carbon bonds exist in the layers, one bound as carbide and the other bound in the form of contamination. The substrate temperature during the deposition process induces the change of the chemical bonds of carbon. Samples prepared at higher temperatures exibit a higher rate of carbide in the layer.

The coating adhesion, one of the most important mechanical properties of thin films, is determined with the scratch test. The surface of the coatings is loaded by a conical diamond tip which is impressed with an increasing normal force ( $\mathrm{dF} / \mathrm{dt}^{-1} \mathrm{Ns}^{-1}$ ) and moved across the specimen with a constant speed $\left(v=0.1 \mathrm{mms}^{-1}\right)$. The acoustic signals, which are generated during the scratch test when flaking of the coating occurs at a critical load, are detected by a 
piezo-electric transducer. The critical load of the coatings deposited at different substrate temperatures is shown in Fig. 9. It is indicated that the adhesion of the VC-coatings increases with the increase of substrate temperature.

The hardness of the coatings was measured with a Vickers indenter at a load of $15 \mathrm{~N}$ (Fig. 10). The hardness values were in the range of $2200-3300 \mathrm{HV}_{0.015}$ and agree with the value $(2900 \mathrm{HV})$ given in literature /7/. However, since the thickness of the coatings is relatively thin (c. $3 \mu \mathrm{m}$ ), the influence of the substrate could not be neglected.

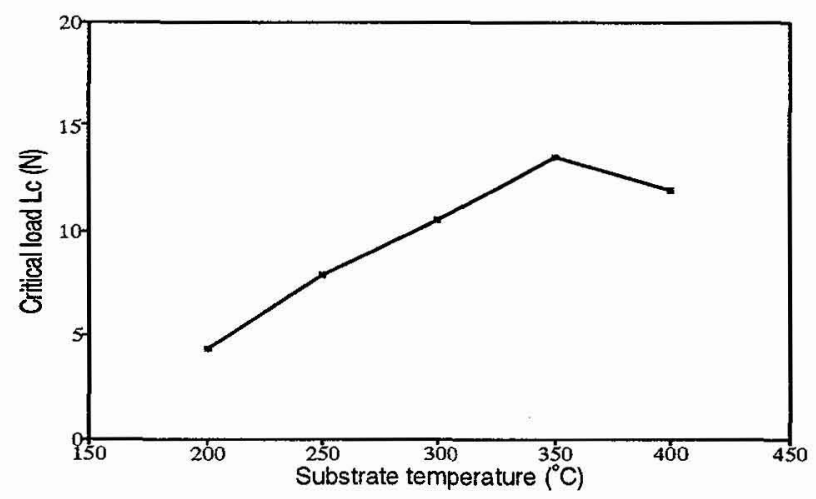

Fig. 9: Critical load of the VC-coatings vs. substrate temperature

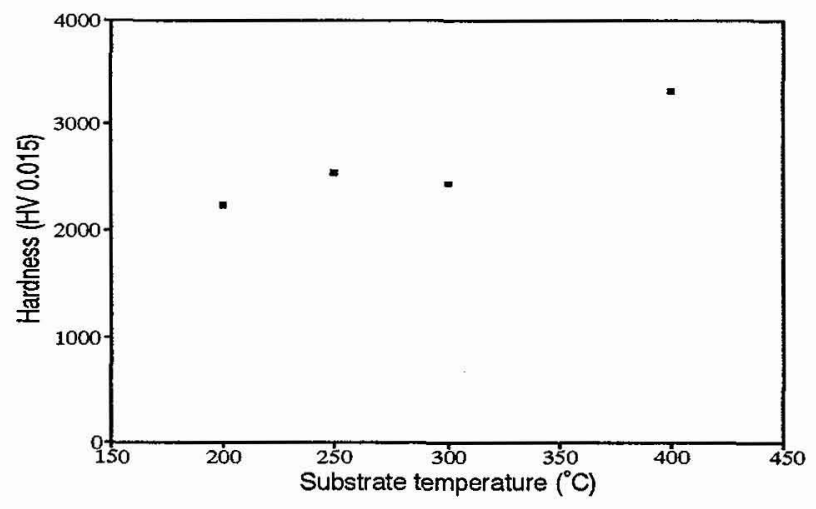

Fig. 10: Vickers hardness of VC-coatings vs. deposition temperature

\section{Discussion}

It is apparent that the deposition temperature influences the growth of the layer in this plasma-assisted MOCVD process. On the one hand the deposition efficiency is independent of the substrate temperature. This could be explained by the thermal stability of the precursor. A homogeneous reaction in the gas phase reducing the deposition rate, which is often observed at elevated temperatures in plasma-assisted MOCVD processes $/ 3 /$, does not appear in the temperature range of $200-400^{\circ} \mathrm{C}$. So the rate determining step in the kinetics of the chemical reaction is the plasma induced decomposition of the metal-organic compound. 
On the other hand, the substrate temperature influences the kind of chemical bonding of carbon in the layer (Fig. 8). For the explanation of the inclusion of carbon in the coatings two points must be taken into account. The first one is the kind of chemical bonding in the MOC. The cyclopentadienyl fragments exhibit a high stability in the glow discharge, so that a lot of carbon can be transported in the gas phase to the exhaust without any further chemical reaction, as could be revealed by mass spectrometry. As a conclusion, this vanadium compound consisting of a metal atom and two stable ligands could be seen as an ideal starting source for a plasma-assisted CVD process for the deposition of vanadium carbide. Secondly, the amount of carbon bound as contamination can be reduced when the temperature is raised. This is a result of the higher desorption rate of the organic by-products from the surface. As a consequence of the enhanced desorption the inclusion of by-products in the growing layer is reduced.

The substrate temperature may also affect the mechanical properties of the layers via the inclusion of organic fragments. The lower critical load of the coatings deposited at lower temperatures can be explained by the adsorption of by-products in the beginning of the process, which disturb the adherence of the layer.

The influence of the substrate temperature on the morphology and the grain size is well known from other deposition techniques and therefore need not to be discussed any more. However, it is shown that the deposition of adherent vanadium carbide films with an acceptable hardness is possible at temperatures below $400^{\circ} \mathrm{C}$.

\section{Conclusions}

Very hard vanadium carbide films can be deposited with the plasma-assisted MOCVD technique at temperatures below $400^{\circ} \mathrm{C}$ when bis(cyclopentadienyl)-vanadium is used as a precursor. Carbon impurities in the layers can have an affection on the mechanical properties like film hardness and adhesion. As the desorption rate of organic fragments from the growing layers can be influenced by the substrate temperature during the deposition process, the amount of contamination of the coatings can be reduced widely. But with optimized parameters the deposition of crystalline, adherent and wear-resistant coatings on HSS is possible without any post heat treatment of the substrate.

\section{Acknowledgement}

We would like to acknowledge the sponsorship of the German Ministry for Research and Technology (BMFT) under contract No. 13N5789.

\section{References}

/1/ T. Arai, J. Heat Treating, 1 (1979) 15-22

/2/ E. Horvath and A. J. Perry, Thin Solid Films, 65 (1980) 309-314

/3/ H.-R. Stock, H. Berndt and P. Mayr, Surface and Coatings Technology, 46 (1991) 15-23

/4/ F. P. Pruchnik, Organometallic Chemistry of the Transition Elements, Plenum Press, New York (1990) 542

15/ J. G. M. Becht, A. Bath, E. Hengst, P. J. van der Put and J. Schoonman, in: M. L. Hitchman and N. J. Archer (eds.) Proc. 8. Europ. Conf. CVD (1991), 617-624

16/ N. Azema, J. Durand, R. Berjoan, J. L. Balladore and L. Cot, in: M. L. Hitchman and N. J. Archer (eds.) Proc. 8. Europ. Conf. CVD (1991), 405-412

/7/ H. Holleck, J. Vac. Sci. Technol. A4 (1986), 2661-2669 